\title{
Erweiterung des Treuhandpartnernetzes im Kanton St. Gallen
}

FMH Consulting Services bietet seit dem 1. Januar 2008 mit der Credor Treuhand AG, Wil, einen weiteren Treuhandpartner in ihrem Netz an.

Seit über 50 Jahren erbringt die Credor Treuhand AG, als Mitglied der Credor Gruppe, mit Erfolg qualifizierte Dienstleistungen in allen Treuhandbereichen. Ein Erfolg, der nicht nur auf Vergangenem basiert, sondern auch auf dem Umgang mit Gegenwart und Zukunft. Wir sprechen von Visionen und dem Mut, die richtige Entscheidung zu treffen. Darum sind wir jederzeit bestrebt, vorhandenes Wissen und Können zu erweitern und mit unserer Kompetenz, Verlässlichkeit und Kundennähe der Kundschaft einen Mehrwert zu sichern.

Durch die gruppeninternen Spezialisten mit entsprechenden Fachausbildungen bietet die Credor Treuhand AG nebst der KMU-Beratung inkl. Lohnadministration, der Wirtschaftsprüfung und der Steuerberatung auch im Bereich Recht den Kunden Dienstleistungen mit Mehrwert an. Seit Jahren begleitet die Credor Treuhand AG zudem Unternehmer und Unternehmungen in der Nachfolgeplanung und -regelung.

Wir erbringen Dienstleistungen mit Mehrwert. Hoffentlich auch bald für Sie. Wir freuen uns auf Ihren Anruf.

\section{Credor Treuhand AG}

FMH Treuhand Services

Roger Hollenstein

Poststrasse 4 - 9500 Wil

Tel. 0719147178 \&ax 0719147179

roger.hollenstein@fmhtreuhand.ch

www.fmhtreuhand.ch

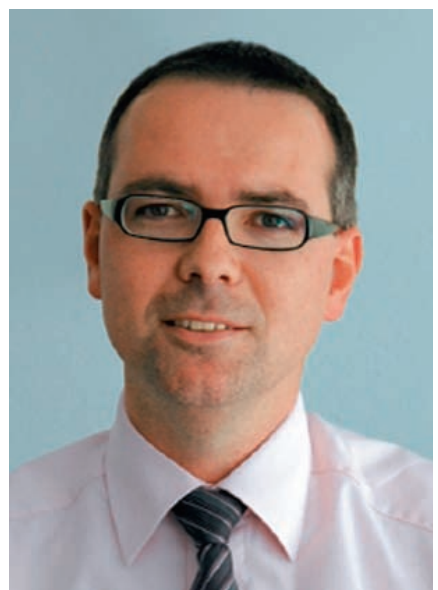

Roger Hollenstein arbeitet seit 11 Jahren bei der Credor Treuhand AG. Nach seiner Tätigkeit auf einem Steueramt erweiterte er seine beruflichen Erfahrungen auf dem Treuhandbereich. Berufsbegleitend hat er sich zum Treuhänder mit eidg. Fachausweis und zum dipl. Treuhandexperten weitergebildet. Er führt seit dem 1. Juli 2007 die Credor Treuhand AG in Wil.

\section{g'FMH TREUHAND}

Praxiseröffnung/-übernahme

Der Weg in die eigene Praxis soll gut geplant sein. Wir unterstützen Sie in folgenden Treuhanddienstleistungen:

- Businessplan, Finanzplan, Finanzierungsmöglichkeiten

- Liquiditätsplanung, Investitionsrechnung

- Buchführung, Kreditoren- und Debitorenbuchhaltung mit Mahnwesen

- Personaladministration und Lohnbuchhaltung

- Abrechnungen mit Sozialversicherungen
- Erstellen von Steuererklärungen

- Steuerberatung und strategische Steuerplanung

- Beratung in Mehrwertsteuerfragen

Vertrauen Sie unserem breit abgestützten Netzwerk an Spezialisten.

\section{FMH Treuhand Services}

FMH Consulting Services $\bullet$ Koordinationsstelle Burghöhe $1 \bullet 6208$ Oberkirch

Telefon $0419250077 \bullet$ Fax 0419210586

mail@fmhtreuhand.ch • www.fmhtreuhand.ch 